\title{
GIS Modelling for Site-Specific Nitrogen Fertilization towards Soil Sustainability
}

\section{Antonis Papadopoulos ${ }^{1}$, Dionissios Kalivas ${ }^{2, *}$ and Thomas Hatzichristos ${ }^{1}$}

1 Department of Geography and Regional Planning, National Technical University of Athens, 9, Iroon Polytehniou Str., 15773 Zografou, Greece; E-Mails: papadop7@survey.ntua.gr (A.P.); thomasx@survey.ntua.gr (T.H.)

2 Department of Natural Resources Management and Agricultural Engineering, Agricultural University of Athens, 75, Iera Odos Str., Votanikos, 11855 Athens, Greece

* Author to whom correspondence should be addressed; E-Mail: kalivas@aua.gr; Tel.: +30-210-5294091; Fax: +30-210-5294092.

Academic Editor: Marc A. Rosen

Received: 22 January 2015 / Accepted: 20 May 2015 / Published: 26 May 2015

\begin{abstract}
Farmers need to make decisions that in most cases incorporate the concept of prediction and can hardly be revoked. One such decision is the application of fertilizing inputs. During past crop management and decision-making on fertilizing practices, many significant errors have been recorded, which have led and continue to lead to reduced production and environmental burden. The methodology followed in this paper involves the use of GIS, fuzzy logic and expert knowledge, in order to model physical processes associated with nitrogen balance in cultivated ecosystems and to evaluate the capabilities of or limitations on the use of certain fertilizers, based on spatial criteria. An original spatial decision support system was designed, developed and applied in a given study area. The system is composed of two modules ("fertilizing rate" and "fertilizing type"), making use of soil, climate and cultivation practices' data, as recorded in the area of interest in quantitative or categorical form. The results of the application spatially classify the involved area according to its demand for nitrogen on the basis of the characteristics of each sub-region. The "fertilizing rate" module suggests reduced fertilizing doses of nitrogenous fertilizers compared to those already applied in the area. The system further divides the area into zones where specific types of fertilizers should be applied, giving a certain prescription for the method and time of application.
\end{abstract}


Keywords: GIS; spatial analysis; site-specific nitrogen fertilization; fuzzy logic

\section{Introduction}

Empirical-, rather than scientifically-justified application of nitrogen $(\mathrm{N})$ fertilizers in various soil conditions was a management reality in Greek farming lands for over forty years. The consequent increasing phenomena of nitrate infiltration to the aquifer led agronomic authorities to take measures against nitrate mitigation and deliver action plans regarding the reduction of nitrate groundwater pollution [1]. Under this context, it is of great importance to look thoroughly into fertilizing plans that comply with certain environmental conditions. These conditions are space and time dependent; making it obvious that parameters, like soil, climate and agronomic practices, should count in every fertilizing plan. Indeed, a successful nitrogen management is one in which the key processes to optimize profitability and environmental protection can be identified, characterized and managed at the appropriate locations and times.

Site-specific nitrogen management (SSNM) refers to the predetermination of appropriate, in terms of space and time, nitrogen prescriptions; in order to increase nitrogen use efficiency and diminish adverse environmental effects [2]. In intensive farming systems, predicting site-specific $\mathrm{N}$ requirements is rather difficult, as processes influencing nitrogen kinetics in soil substrate, like plant uptake, mineralization, immobilization, denitrification, leaching and others, interact in complex ways [3]. Moreover, as soil and weather conditions, tillage practices and the dynamics of crop growth influence various components of the N-cycle, the complexity of controlling nitrogen rises [4].

As mentioned, there is a demand for the development, calibration and validation of fast-working tools that can assess $\mathrm{N}$ fertilizing demands that do not burden the environment with nitrate concentrations in groundwater resources. Various simulation models have been developed and evaluated in the field of nitrogen management [5]. Many of them have been also linked with spatial modules, enabling fertilizing zone mapping [6,7]. The majority of these models require a plethora of data, which in many cases are not easily retrieved. Moreover, their evaluation procedure requires specific knowledge and can be regarded as demanding and complex in terms of computer handling skills.

A crucial component of SSNM is spatial analysis realized through the use of geographical information systems (GIS). Agricultural management interacts with environmental parameters and natural resources that have an obvious spatial character, and hence, GIS are crucial in enhancing agriculture production, especially with reference to field fertilizer application [8]. In most studies, GIS are usually employed to process model inputs and to visualize results. In order to solve more sophisticated and special problems, like that of fertilizing management, enhanced spatial modelling is needed, in the form of integrated spatial models, which ought to couple and integrate GIS with robust decision methodologies. In classical GIS modelling, multiclass maps are usually converted into binary predication patterns, in which crisp boundaries between areas are assumed. Reality though never involves precise and certain boundaries. As stated in [9] from the cybernetics point of view, nitrogen management is a typical "control situation under uncertainty". Furthermore, from the environmental point of view, the nitrogen cycle is typically surrounded by vagueness and inherent uncertainties, as it involves many physicochemical processes and 
parameters, which are difficult enough to be modelled under the sharp limits of a mathematically crisp logic. Fuzzy logic is a serious candidate for supporting decisions in environmental phenomena, as it introduces subjectivity and vagueness in an analysis and for this reason is incorporated into the present study. Various research works reveal the effectiveness of fuzzy theory in decision problems concerning fertilizing crops $[10,11]$.

The objective of this study is to systemize and automate the methodological steps needed towards the identification and map production of discrete nitrogen fertilizing zones for cotton crop. Further, through the design, development and application of a spatial decision support model, we aim to demonstrate that GIS and fuzzy theory can be of valuable help for decision makers in order to delineate agricultural areas according to their actual fertilization needs complying with SSNM. It is envisaged that the development of such a spatial tool will be of valuable help for external agronomic services towards prescribing accurate site-specific fertilizing guidelines, benefiting the producers and the environment.

\section{Materials and Methods}

\subsection{System Design}

A justified decision on fertilizing practices ought to be based on scientific knowledge and experimental results ideally combined with agronomic experience and local environmental conditions. Nitrates in soils consist of a very mobile form of nutrient, making its application a rather demanding and multilevel decision. In order to propose integrated and realistic nitrogen fertilizing recommendations, aspects, like the dose of the proposed fertilizer, its type, meaning the form of the contained nitrogen ions, the number of doses, the time and method of the application and the spatial distribution of the suggested fertilizer quantities, should be considered. The latter is rather imperative as long as we accept the agricultural environment as a continuous, but ever-changing system influenced by soil, topographic, climatic and agronomic factors.

The proposed methodology considers two system spatial modules. The first one undertakes the computation of the fertilizer rates, which are proposed for application during a cultivation period (fertilizing rate), and the second delivers descriptive recommendations regarding the type, time and method of fertilization (fertilizing type). The structure of the rate module is based on the simulation of the nitrogen cycle in agronomic systems, as it is "translated" via the nitrogen balance equation. The type module takes into account two risk criteria: the avoidance of soil degradation due to acidification and the restriction of nitrate leaching losses [12]. Both modules integrate fuzzy logic concepts and functions under a GIS environment, meaning that they are designed to function using digital maps and relational geodatabases. Moreover, the system can support decision making regardless of certain scales and is adjusted to end users' needs and data availability. The architecture of the spatial decision support model is presented in Figure 1. The modules' development was made under the Model Builder 10.1 tool environment of the ArcGIS software (ESRI Inc., Redlands, CA, USA) and takes advantage of its capabilities in spatial analysis. 


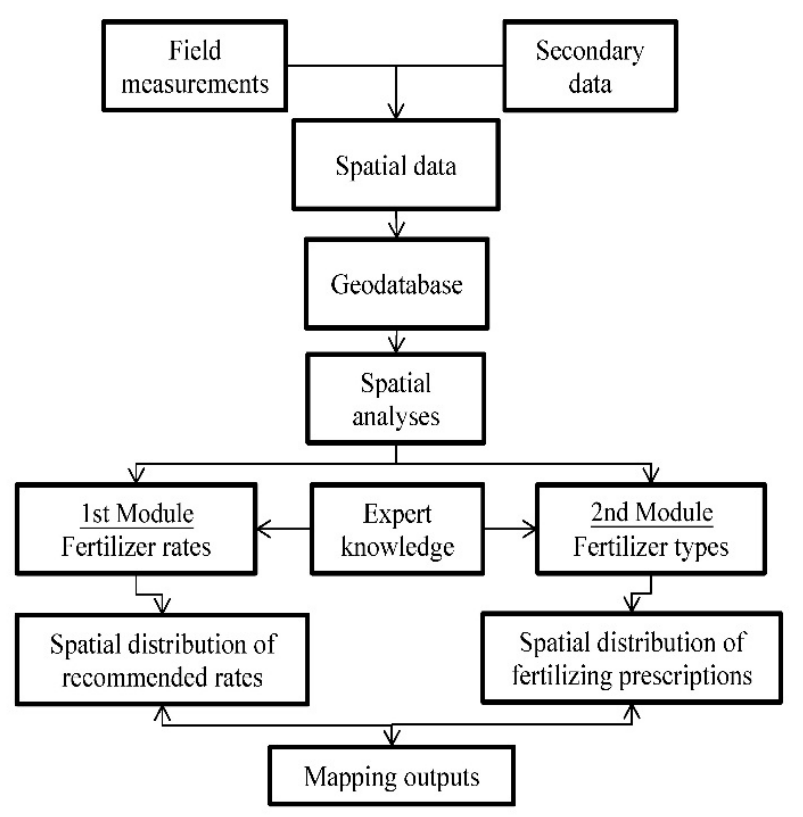

Figure 1. Conceptual system design.

The variables incorporated into the system and the data used for the system evaluation were carefully selected according to their scientific and proven correlation with the objectives of a rational and an environmentally-compliant fertilizing scheme. Moreover, the ability of accessing and retrieving the modules' data in combination with the least possible number of parameters were crucial criteria for selecting the system's variables. The system remains flexible, as one can add variables or dismiss redundant ones, according to site-specific needs. Data can be spatial or descriptive, continuous or categorical. For the level of expert knowledge, the system provides, through the use of fuzzy logic, a robust framework for representing and dealing with vague environmental phenomena.

\subsection{Spatial Modules "Fertilizer Rate" and "Fertilizer Type"}

The "fertilizer rate" module is based on the fuzzy approximation of the nitrogen equilibrium [13]. The module classifies the reference area according to the soils' needs for nitrogen resulting from the fuzzy calculation of nitrogen processes. The processes accounted for in the module are nitrate leaching, denitrification, plant uptake and ammonia volatilization, which are considered as outputs from the soil system, and the according soil system inputs, meaning organic nitrogen mineralization, inorganic residual nitrogen and nitrogen added through irrigation water. Nitrogen immobilization was not considered in the module, due to its low contribution to the equilibrium compared to the other outputs under Greek soil conditions. Through the fuzzy reasoning method, the module identifies those locations that have the most need for nitrogen fertilizers, classifying the reference area between a -1 and 1 value with respect to this "need". In other words, negative values refer to sites where nitrogen inputs are less than the outputs, and for those areas, the module proposes the application of fertilizer. Positive values suggest the adequacy of nitrogen reserves, and the only fertilization scheme proposed should target preserving nitrogen availability throughout the cultivation period. The "fertilizer rate" module requires 14 state variables in order to be successfully evaluated (Table 1). 
Table 1. Parameters in use by the two sub-modules.

\begin{tabular}{|c|c|c|c|}
\hline Category & Parameter & Fertilizer rate & Fertilizer type \\
\hline \multirow{6}{*}{ Soil data } & Soil inorganic nitrogen (inorganic) (ppm) & $\sqrt{ }$ & \\
\hline & Soil acidity $(\mathrm{pH})$ & $\sqrt{ }$ & $\sqrt{ }$ \\
\hline & Soil organic matter content (om) (\%) & $\sqrt{ }$ & \\
\hline & Clay content percentage (clay) (\%) & $\sqrt{ }$ & $\sqrt{ }$ \\
\hline & Soil moisture (moist) $(\%)$ & $\sqrt{ }$ & \\
\hline & Soil drainage (drainage) (well/poorly drained) & $\sqrt{ }$ & \\
\hline \multirow{2}{*}{ Climate data } & Mean soil temperature during cultivation period (temp) $\left({ }^{\circ} \mathrm{C}\right)$ & $\sqrt{ }$ & \\
\hline & Mean total rainfall during cultivation period (rain) $(\mathrm{mm})$ & $\sqrt{ }$ & $\sqrt{ }$ \\
\hline \multirow{6}{*}{$\begin{array}{l}\text { Agronomic } \\
\text { data }\end{array}$} & $\begin{array}{l}\text { Whether the producer makes use of acidifying fertilizers } \\
\text { (type) (yes/no) }\end{array}$ & $\sqrt{ }$ & $\sqrt{ }$ \\
\hline & $\begin{array}{l}\text { Whether the producer incorporates the fertilizers } \\
\text { (incorporation) (yes/no) }\end{array}$ & $\sqrt{ }$ & \\
\hline & Yield potential (yield) $(\mathrm{kg} / 0.1 \mathrm{ha})$ & $\sqrt{ }$ & \\
\hline & Quantity of irrigation water (quantity) (m³/0.1 ha) & $\sqrt{ }$ & \\
\hline & $\begin{array}{l}\text { Concentration of inorganic nitrogen in irrigation water } \\
\text { (quality) (ppm) }\end{array}$ & $\sqrt{ }$ & \\
\hline & Irrigation system in use (system) (sprinkler/drip) & $\sqrt{ }$ & $\sqrt{ }$ \\
\hline
\end{tabular}

The "fertilizer type" module makes use of 5 state variables (Table 1) and considers two soil degradation risks in order to propose a nitrogen fertilizing prescription. The first one is soil acidification risk and is fuzzy calculated with the contribution of soil $\mathrm{pH}$, mean annual rainfall, the irrigation system in use and the use of acidifying fertilizers. The second risk refers to nitrate leaching potential, which is fuzzy controlled by soil clay content and mean annual rainfall. The specific module fuzzy calculates the two risks and ascribes membership values ranging from 0 to 1 for the vulnerability of locations to those risks. Increasing membership values indicate areas with subsequently increasing risk of leaching and acidification potential. The module further maps certain fertilizer recommendations in the form of prescription codes to the previously-generated fuzzy categories. The latter assignment was based on scientific and applied judgement as elicited through interviews and participation of experts.

\subsection{Study Area}

The area that was chosen for the application and verification of the spatial fertilizing model concerned various cotton growing lowlands in the plain of Kopaida (Viotia Prefecture), central Greece $\left(38^{\circ} 25^{\prime} \mathrm{N}: 23^{\circ} 06^{\prime} \mathrm{E}\right)$ (Figure 2). The choice of the study area was made based on environmental criteria. The plain of Kopaida is well known for its cotton fields and also for constituting a substantial area of nitrogen fertilizing applications. The plain is also regarded as one of the seven Greek regions that are most susceptible to nitrate pollution. Subsurface waters in the region appear to suffer from high values of nitrate concentrations, caused by the intensification of agriculture and the irrational use of nitrogen fertilizers. Moreover, the study area is spatially surrounded by an ecologically-sensitive zone, marked by the network Natura 2000 (http://ec.europa.eu/environment/nature/natura2000/index_en.htm) as a habitat of significant interest that must be safeguarded and preserved. The region in which the study fields are located covers around 9000 hectares. 


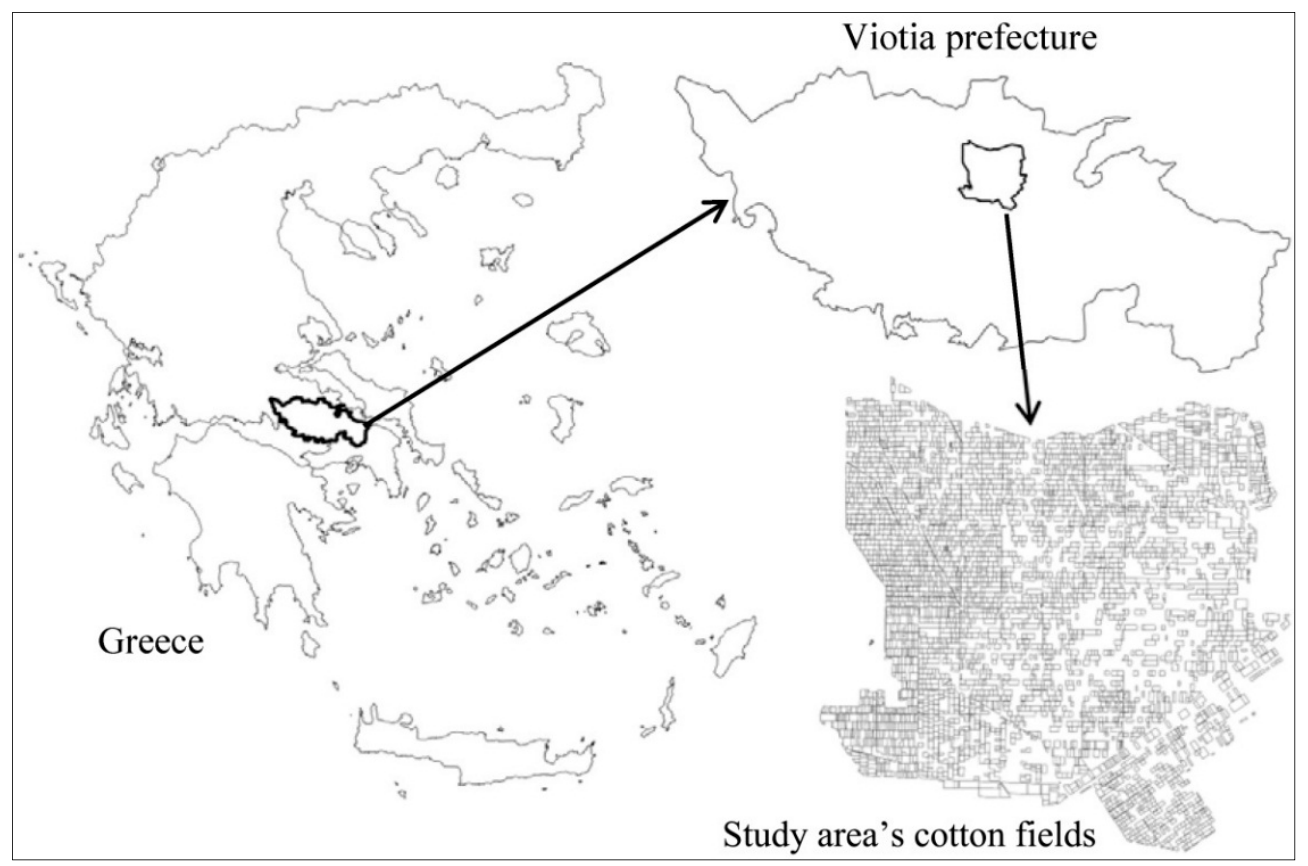

Figure 2. The study area.

The average length of the study area is $11 \mathrm{~km}$, while its width is about $8 \mathrm{~km}$. The average altitude (above sea level) is $95 \mathrm{~m}$. Regarding the terrain of the region, it is smooth with very small slope values. The soils of the wider area have developed on calcareous or non-calcareous parent materials in areas with large to very small slopes (area of interest) under conditions of intense erosion of native materials or materials recently transferred. The spatial distribution and classification of the reference soils are presented in Figure 3 [14].

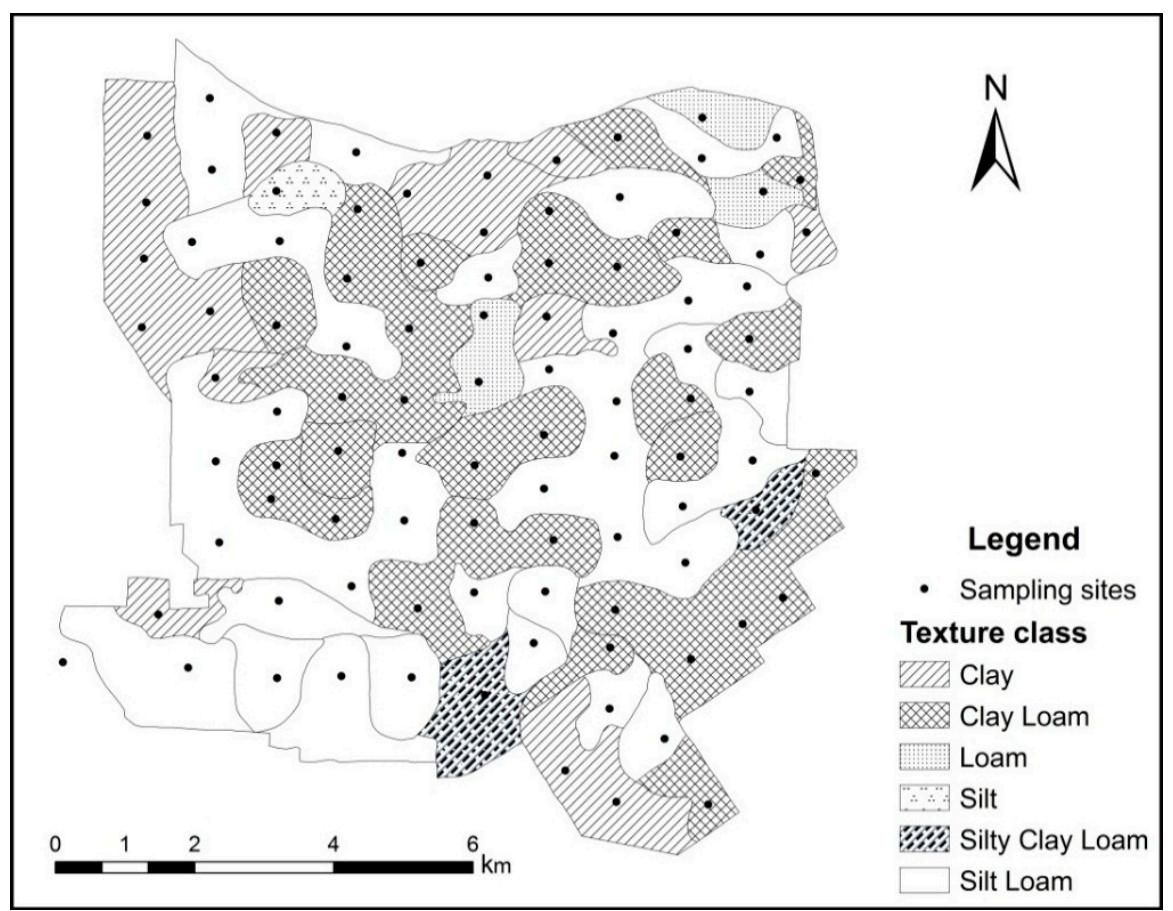

Figure 3. Soil texture map of the study area and the soil sampling sites. 
The climate of the basin, part of which is the area of interest, is Mediterranean with continental climate characteristics at higher altitudes, and it is ranked at the semi-dry climate zone. Across the wider region, there are significant changes in climatic parameters. Mean annual rainfall reaches $678 \mathrm{~mm}$, $45.1 \%$ of which occurs during winter months. In summer, mean rainfall is limited to $50 \mathrm{~mm}$. The warmest month appears to be July, characterized by a mean air temperature of $26.2^{\circ} \mathrm{C}$, while the coolest is January $\left(6.6^{\circ} \mathrm{C}\right)$. With regard to frost, this appears mainly during the months of December, January, February and rarely in March, often causing damage to crops.

The main crops cultivated in the region are cotton, cereals, olives, corn, vines, tomato, onions, alfalfa, potatoes and various other vegetables. According to existing data, the area is considered as one of the main cotton production areas in the country [15].

\subsection{Data Collection and Management}

Soil physicochemical parameters used in the study were retrieved from an existing soil survey of the area [14]. In order to validate the rather outdated data contained in the soil survey, 95 soil samples across the area were taken and analyzed for the basic physicochemical properties. Certain differences concerning mostly the surface soil layer were realized and accordingly adjusted to the updated data results. More specifically, the soil survey was digitized, producing a polygon layer consisting of soil mapping units and a point layer presenting the positions of soil samples. Descriptive data for the point layer were analytical values for top soil inorganic nitrogen, soil $\mathrm{pH}$, organic matter content, clay content and soil moisture, while for the polygon layer, they were the soil drainage categories. Soil parameters in areas where no data were available were estimated with the use of the spatial interpolation method, kriging [16]. The method is based on statistical models that include autocorrelation, meaning statistical relationships among the measured points, and it was chosen because it not only has the capability of producing a prediction surface, but also provides some measure of the certainty or accuracy of the predictions. Further, the number of the points was quite enough for the interpolation method to produce reliable results.

Climate data used in the module were mean soil temperature and mean rainfall during cotton cultivation period. Both parameters were retrieved from a network of 15 weather stations for a 30 -year reference period. The positions of the stations were digitized, comprising a point layer, and in order to produce estimated continuous value surfaces across the study area, the Thiessen polygon method was applied. Although kriging is the suggested interpolation method concerning meteorological data [17], the limited number of operational weather stations in the area and the fact that most hydrological models make use of the Thiessen polygon methodology led the writers to choose the latter for interpolating soil temperature and rainfall parameters.

Agronomic data used in the module referred to those management actions that a producer performs during a growing period and are related to irrigation and fertilization (Table 1). In this category, the yield potential was included, as it is majorly affected by management decisions. Data were collected and managed at the field level; hence, the spatial reference of these data was the fields' boundaries. The methodology followed for field data acquisition was the compilation and submission of 50 questionnaires, directed and completed by the according number of local growers. The respective descriptive data 
attached to field polygons and collected through the questionnaires regarded the area of the fields, seeding data, applied fertilizers, former fertilizing strategies, irrigation plans and yield quantities.

All datasets were stored in a geospatial database (Figure 4). Continuous point data, like soil acidity, inorganic nitrogen, climate parameters, etc., after being spatially interpolated, were transformed into raster data structures with a cell size of 10 meters. Soil drainage classes, which were derived from the soil survey as a polygon layer, were directly converted to a raster surface under a 10-meter cell size. Additionally, agronomic data (continuous and categorical), after being attributed to polygon field features, were also converted to raster grids. The fuzzy logic method was chosen to further elaborate the resulting continuous raster files.

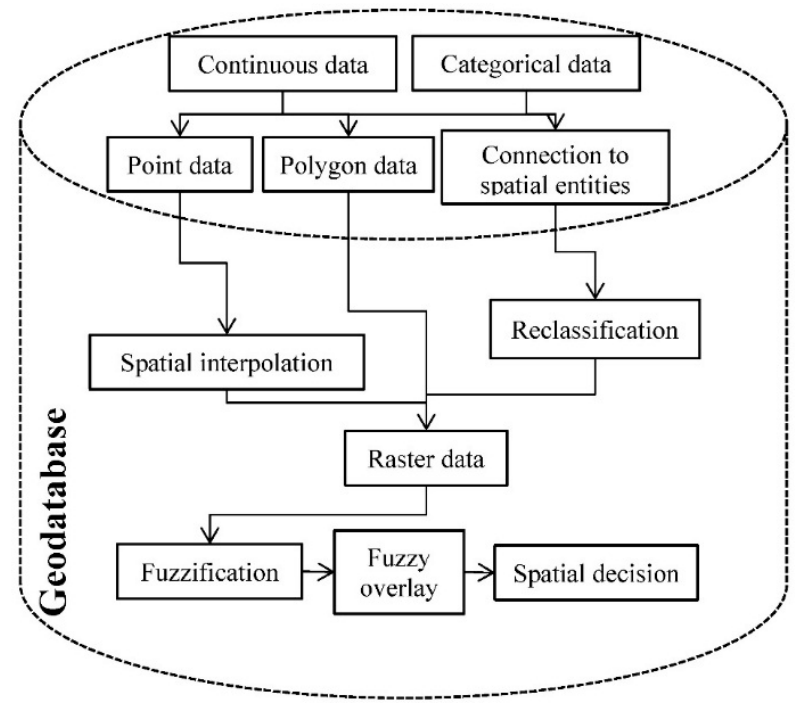

Figure 4. Data and processes flow chart under the geodatabase environment.

\subsection{Sensitivity Analysis}

The use of models, particularly in the environmental domain, inevitably involves the presence and treatment of uncertainties. In general, when a system is used to simulate a complex process, the application of sensitivity analysis becomes particularly important for both the reliability of the model and its usability [18]. The sensitivity analysis is an integral part in the processes of decision-making, considering that it can identify the effects on the performance of a decision support system, when fluctuating one or many of its structural parameters [19].

In order to identify the most important input parameters, as derived from the two developed models, a local sensitivity analysis method that takes a one-at-a-time (OAT) approach was implemented. Performing this approach, the impact of changing values of each non-categorical model parameter on the models' outputs is evaluated one at a time. In other words, the model output responses are determined by sequentially varying each of the model parameters and by retaining all other parameters at their "base" values. The "base" values refer to the initial parameter values under which the two modules were evaluated to produce the base model outputs and with which all sequential runs were compared. The quantification of the sensitivity analysis results was expressed by a dimensionless index (Sensitivity Index I), which was calculated as the ratio between the relative change of model output and the relative change of a non-categorical parameter [20]. 
The parameters that were taken into account in the sensitivity analysis for the "fertilizing rate" module were: soil inorganic nitrogen, clay content, $\mathrm{pH}$, organic matter, soil moisture, mean soil temperature, mean rainfall, quantity of irrigated water, inorganic nitrogen in irrigated water and yield. A sensitivity approach was also followed in the case of the "fertilizing type" module, where the parameters involved were clay content, $\mathrm{pH}$ and mean rainfall. As the output of the "fertilizing type" module is categorical in the form of prescription codes, the corresponding sensitivity measurement was the percentage of fields (expressed in area units) for which the prescription code differed from the base line, after the application of each variation in the initial values.

\section{Results and Discussion}

The fuzzy methodological approach followed concerned two discrete steps. Firstly, the raster datasets were converted into fuzzy evidence maps via specific membership functions, which transformed raw data into fuzzy sets having values between zero and one. Regarding categorical raster layers, those underwent reclassification in order to gain values at the range of zero to one.

The choice of membership functions for fuzzifying all parameters involved in the two models was based on the semantic import model [17]. Accordingly, the specific membership functions used to "map" each parameter's range value to zero to one space were directed by the effect that each parameter had on the controlling process. Indicative membership functions used in each module are presented in Figure 5a-e. At the left side of the figures, the mathematical equations and the corresponding graphs are presented, while at the right column, the mapping results of the fuzzification procedure are cited.

At this modelling stage, GIS layout capabilities offer representations of the degree of contribution each input variable has on nitrogen balance parameters along with their spatial distribution across the study area. The color symbology presents the membership grading that a state variable has on each nitrogen balance parameter. In Figure 5a, low clay content values, as presented by the soil types in Figure 3, are linked to high membership values of leaching potential, which are symbolized with green colors in the map at the right column. Further, the relationship between residual nitrogen and inorganic nitrogen concentration in soil was ascribed with a linear function positively sloped (Figure $5 b$ ), translating that high inorganic $\mathrm{N}$ in soil directly leads to increased residual nitrogen values. In addition, nitrogen mineralization, which is considered as an input process to the soil system, is partially affected by organic matter content in a positive way, meaning that high organic matter values with a low $\mathrm{C} / \mathrm{N}$ ratio advance mineralization, enriching soil with nitrogen inorganic forms. In this case, a sigmoidal function, in which the larger the values of organic matter, the more likely the referenced sites are to be members of the "large" mineralization set, was applied (Figure 5c). Moreover, when full membership to a fuzzy set ranged between a specific value, a Gaussian or a triangular function was used. In this case, regarding the mineralization "large" set, sites with soil $\mathrm{pH}$ around neutral or slightly alkaline would have high (near one) membership values (Figure 5d). Nitrogen mineralization is a rather complex process concerning various inaccuracies stemming from soil characteristics and is usually quantified through deterministic models [21,22]. In the present study, the fuzzification scheme used allows a more structured way of dealing with such uncertainties through the partial inclusion (membership) in one or more linguistic sets. In Figure 5e, another linear function is presented mapping the effect of yield potential in plant nitrogen uptake. Sites with high yield potential are more likely to provide more nitrogen 
compounds to cotton plants, given the weather and soil conditions, increasing nitrogen plant uptake, meaning extra losses from the soil system. Triangular-shaped functions are characterized by their simplicity and their ability to simulate every other non-triangular function [23] and, hence, were integrated into the methodology followed.
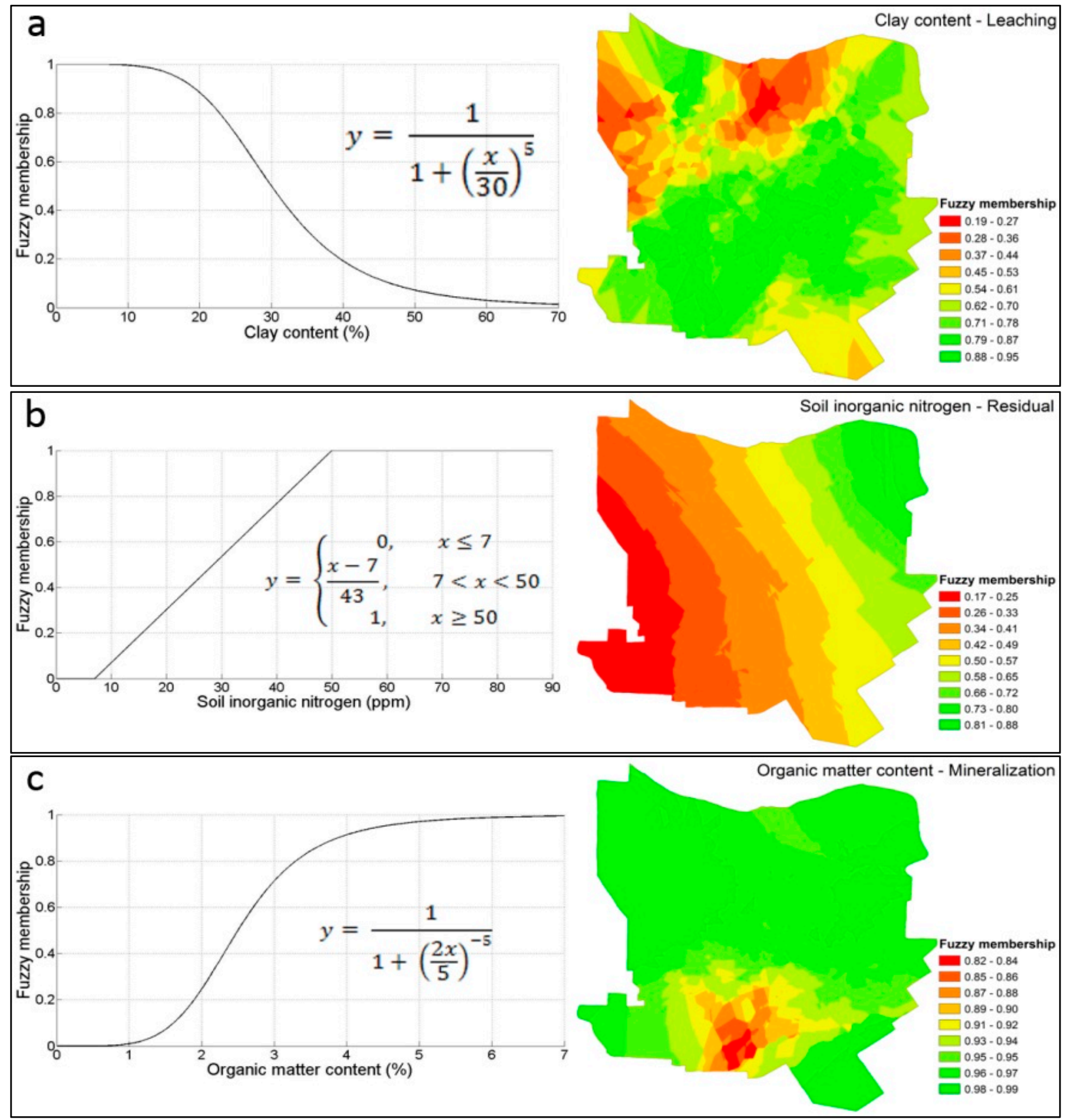

Figure 5. Cont. 


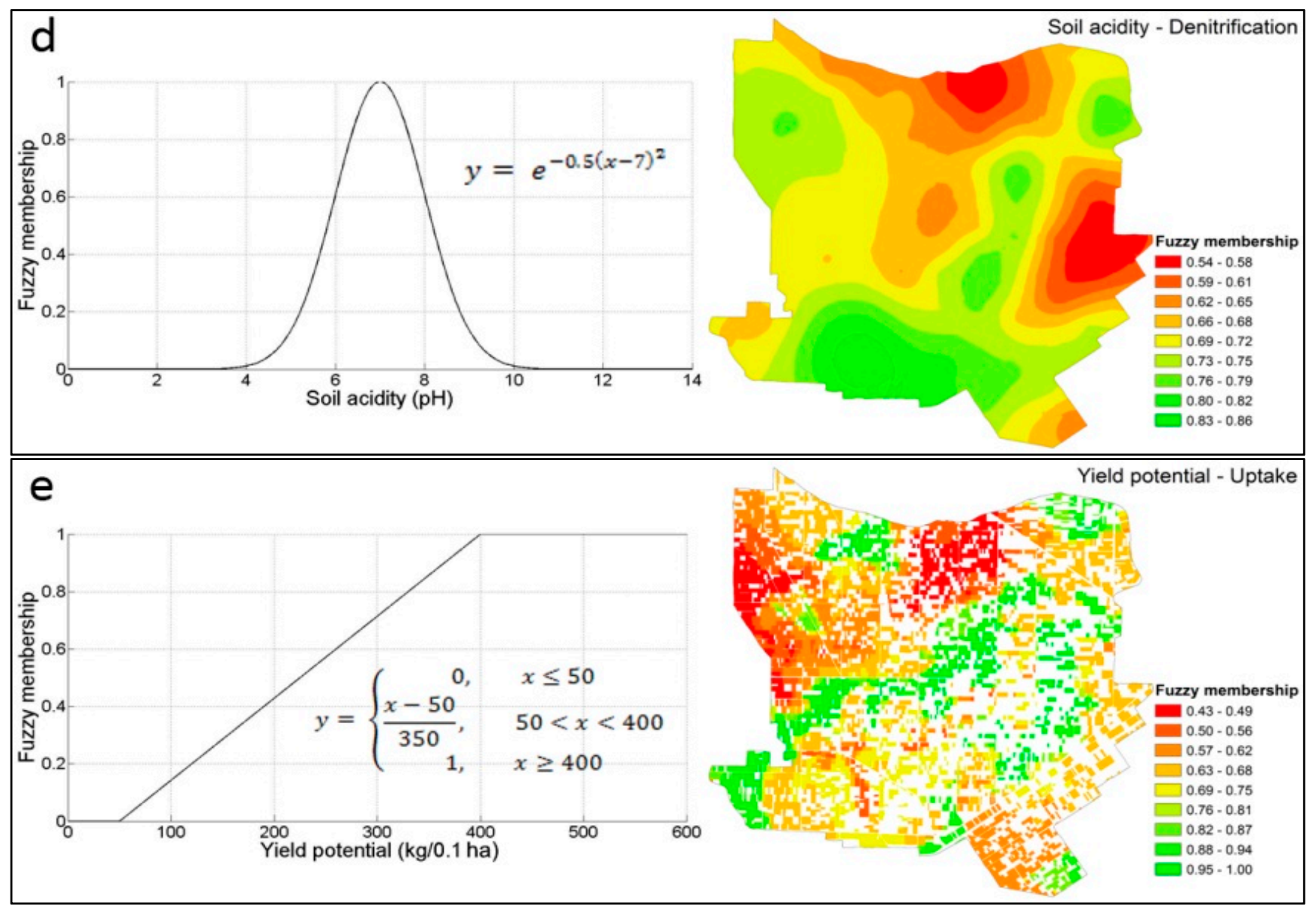

Figure 5. Fuzzy membership functions coupled with GIS mapping representations in the study area, concerning various inputs and nitrogen balance parameters of the system. Red to green color implies stronger membership of the fuzzified variable to the modelled balance parameter. (a) Clay content-Leaching potential; (b) Soil inorganic nitrogen-Residual nitrogen; (c) Organic matter content-Mineralization; (d) Soil acidity-Denitrification; (e) Yield potential-Nitrogen uptake.

The fuzzy overlay operator used in the levels of the two modules was "AND". The linguistic interpretation of a fuzzy operator is related to the specific decision problem and the fuzzy inference procedure in use. Additionally, the choice of the proper connecting fuzzy operators can be evaluated and altered a posteriori after successive implementations of a fuzzy system. In the present study, the ascription of the logical operator "AND" is fulfilled through the minimum function, which responds to the intersection of fuzzy sets [24]. In general, the intersection of fuzzy sets ("AND" operator) is most commonly used in a fuzzy rule-based evaluation. Intersection accounts for the compatibility between two or more fuzzy sets and their range values in order to ascribe truth membership values. The minimum value of these values determines the degree that these sets are being intersected [25]. Except for the "AND" operator, during the development of the system, various weighted operators were tested, like "SUM", "PRODUCT" and "GAMMA". However, these operators tended to balance and diminish various differentiations in raw and intermediate data, resulting in homogeneous spatial patterns. 
Mostly, model-oriented research on simulating nitrogen balance that proposes fertilizing rates and prescriptions is based on existing or modified simulation models, which are usually evaluated and validated in various study areas concerning a variety of crop, soil and weather conditions. The majority of such studies compares model simulations with experimental field results with significant findings regarding model usefulness in planning fertilization schemes [26,27]. The modules presented here integrate expert knowledge, the elicitation of which was realized through dialogue-based procedures with experts (producers, researchers, academics) in the fields of cotton cultivation and nitrogen fertilizing. System development is overall based on expertise acquisition, which is "translated" via fuzzy logic into fuzzy inference and modelled in a GIS environment with no in situ experimentation. Through ArcGIS's model builder environment, the modules' evaluations are automated by linking data input, GIS tools/functions and final outputs. In other words, all processes can be saved and models can be rerun at any time, which is particularly useful when adjustments have to be made in the analyses. Further all cartographic results of the models (intermediate and final) can be illustrated, making the modelling evaluation a transparent process. This is very helpful at the model development stage, as one can simply change one parameter, rerun the model and see the new results, rather than redoing the entire analysis. Moreover, it enforces the flexibility of the entire system when adaptation and evaluation of the modules to new case studies are involved or when various scenarios are being explored.

The "fertilizing rate" and "fertilizing type" modules of the system are presented in Figures 6 and 7, respectively. In both modules, the containing model elements can be summed into three categories: variables, tools and connectors (ESRI Inc.). Variables are model parameters that hold a specific value or a reference to data stored on disk. In the referenced modules, input variables are depicted in blue color, and derived variables are colored in green. The "tools" elements are the basic building blocks of the modules' workflows. These geoprocessing tools perform various operations on geographic or tabular data contained in the modules and are presented with yellow rectangles. Lastly, connectors undertake the connection between variables and tools. The connector arrows show the direction of processing, which in Figures 6 and 7 is heading from left to right. 


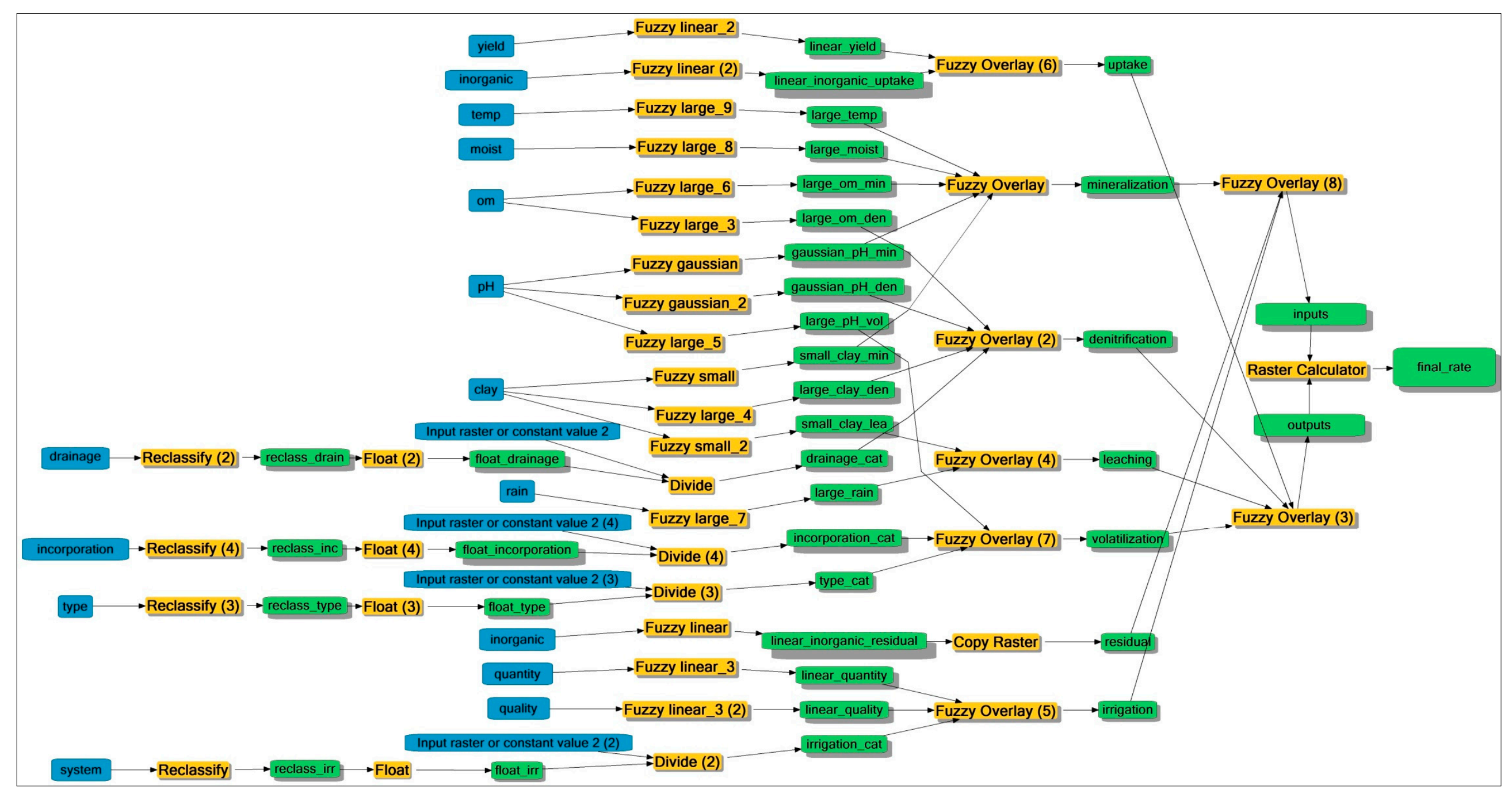

Figure 6. The "fertilizer rate" module presenting the data flow, transformations and final output. Input variables are presented in blue colors, derived variables in green and geoprocessing tools in yellow. 


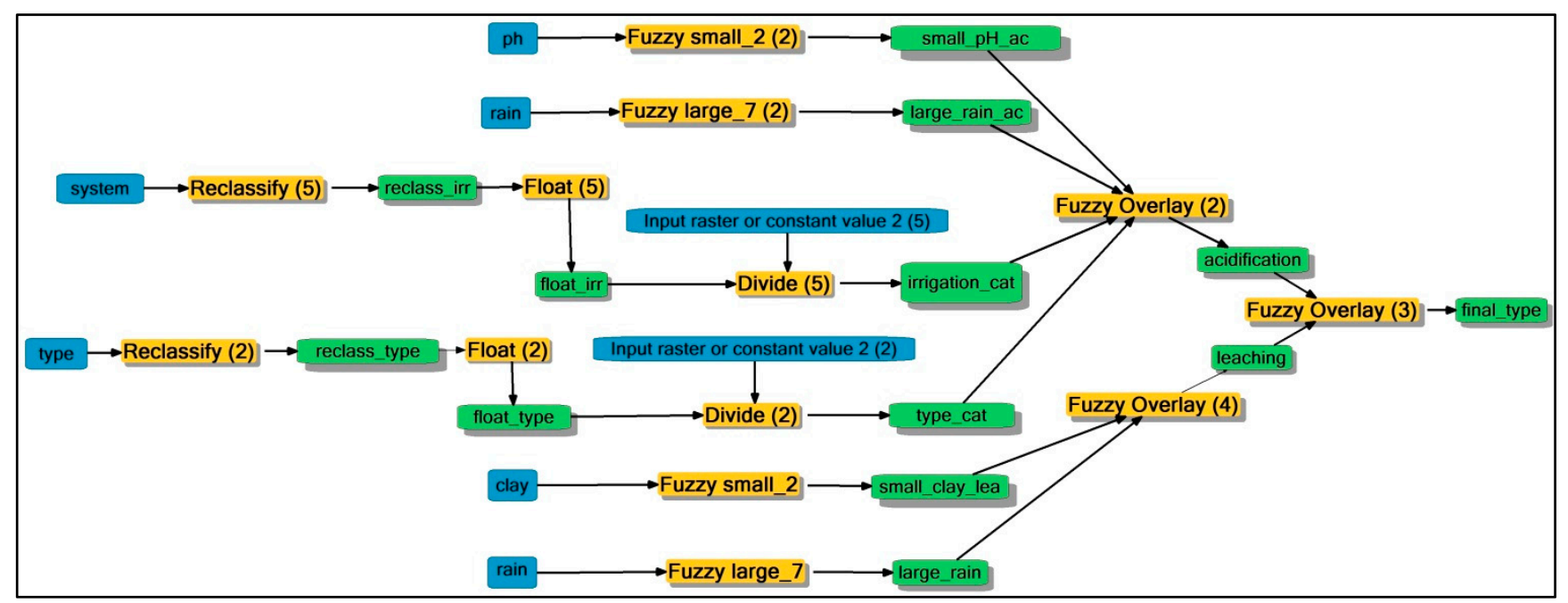

Figure 7. The "fertilizer type" module in Model Builder view. Input variables are presented in blue colors, derived variables in green and geoprocessing tools in yellow.

For the "fertilizing rate" module (Figure 6), three conceivable levels of data processing can be identified. The first one delivers the conversion of all data into floating-point raster format (floating point raster data store numbers with a decimal portion as the values of each cell) and introduces the fuzzification method of their value ranges. Under this level, continuous variables are directly fuzzified using “fuzzy small”, "fuzzy large", "fuzzy linear" and "fuzzy Gaussian” membership functions [28,29], while categorical data undergo a pre-processing step. This step presupposes the use of reclassification in order to attribute numeric values to string ones (reclassify tool) and to further divide the result by a factor to normalize the output values to be between zero and one (divide tool). The second level performs the fuzzy overlay methodology, which groups the fuzzified parameters into the nitrogen balance factors and, in a further step fuzzy, calculates the nitrogen inputs and outputs. The last level performs the mathematic subtraction between inputs and outputs and yields the final classification of the study area into fertilizing zones. Accordingly, the "fertilizing type" module is composed by two thematic levels (Figure 7). The first one involves the fuzzification of input parameters and the second their fuzzy overlay. In this case, the final computation of the total risk against soil sustainability is accomplished through the SUM fuzzy operator, aggregating the leaching and acidification risks into an escalating membership value ranging from zero to one.

The evaluation of the "fertilizing rate" module in the study area at the beginning of the cotton cultivation period (prior to seeding) produced a classification of the area into continuous values ranging from -0.69 to 0.35 (Figure 8). The value range involved in the color spectrum originated from the algebraic subtraction of the nitrogen inputs minus the according outputs. According to the module's results, many sites (yellow to red hues) are attributed with negative values, meaning that for those fields, the outputs of nitrogen equilibrium exceeded the input ones. The area covered by those sites reaches $81.1 \%$ of the total area examined. Under the site and time-specific conditions of the respective evaluation of the module, the area under negative membership values needs to be fertilized with nitrogenous fertilizers. The cartographic approach presented offers the per field or even sub-field computation of fertilizing rates by multiplying each pixel value contained in a field with the fertilizing dose that is proposed by the Greek Action Plan for the mitigation of nitrates in water resources of the vulnerable 
district of Kopaida [30]. In essence, Figure 8 grants the study area with a weighting factor under which each producer can calculate his field's nitrogen needs and further provides a site-specific refinement in previous catchment level fertilizing suggestions, which were presented in the referenced Action Plan. Various research studies have delineated regions into management zones concerning soil fertility indicators using spatio-statistical clustering methods [31,32]. Their results indicate that the identification of distinct soil management zones can optimize fertilization scheduling and diminish environmental risks. Management zone delineation in the presented system originates from modelling nitrogen equilibrium using fuzzification and fuzzy overlaying procedures, and it is expert knowledge oriented, rather than data driven. Furthermore, the system is entirely built under GIS software taking advantage of its integrated fuzzy capabilities as a means of managing the integral uncertainty of the environmental phenomena modelled.

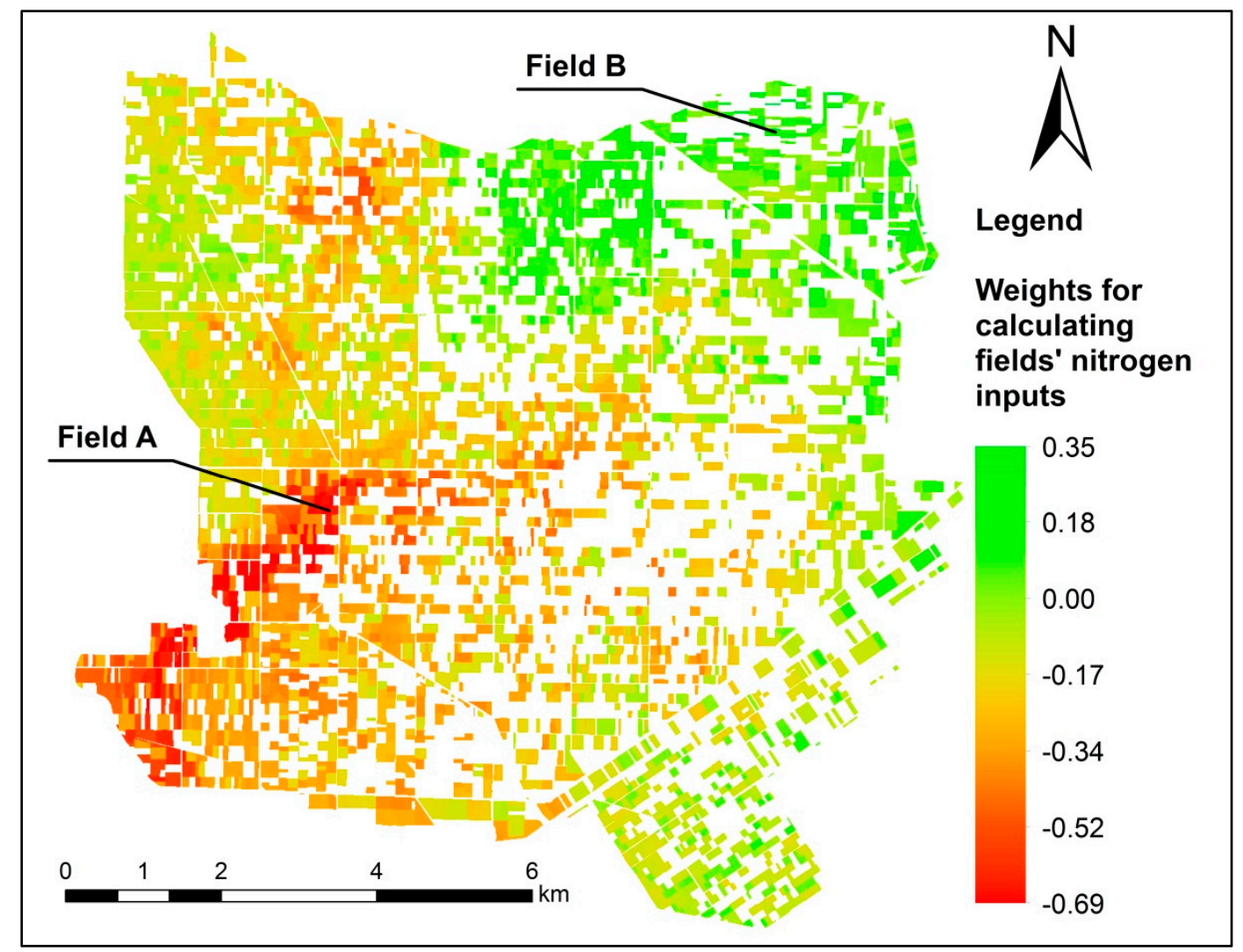

Figure 8. The spatial output of the "fertilizing rate" module.

The sites attributed with positive values account for $18.9 \%$ of the total examined area, and according to the module's design and evaluation, the corresponding fields should not need any nitrogen fertilizing inputs prior to or during seeding. However, for those sites, nitrogen fertilization should concern surface applications during the cultivation period adapted to plant needs. The sites needing no or very low fertilizing rates are mainly characterized by high concentrations in inorganic nitrogen, clay particles and organic matter combined with low yield potential. Comparing the characteristics of two fields situated in different parts of the study area, for which the module attributed different weights (Figure 8), the reasoning of the outcome is revealed. For example, Field A is proposed to be fertilized with rather high amounts of nitrogenous fertilizer (weight factor: -0.65 ) as opposed to Field B, which is advised not to 
be fertilized at all (weight factor: 0.30). Indeed, Field A is situated in an area where, according to input data, texture is medium to coarse, soil is well drained, organic matter content is low compared to the average of the area and the levels of inorganic forms of soil nitrogen are also low. These soil conditions favor nitrate leaching, which, in combination with cultivation and irrigation practices, like the absence of fertilizer incorporation and the use of sprinkler irrigation systems, increase nitrogen losses. Consequently, the "fertilizing rate" module fuzzy simulating the relations between those characteristics and the nitrogen balance's parameters suggests increased fertilizer inputs. On the other hand, in Field B, soil retains more fertile properties in terms of inorganic nitrogen and organic matter content, while the producer uses a drip irrigation system, which reduces nitrate losses. Moreover, the second producer incorporates the applied fertilizer, a practice that diminishes ammonia losses through volatilization. In the case of Field B, the module fuzzy integrates the underlined conditions and proposes minimum fertilizer doses. The aforementioned fields are set two rather extreme cases regarding fertilizing recommendations; however, the module manages to prescribe weighted doses to all cotton fields in the study, through the use of fuzzy inference methodology.

Overall, the results indicate reduced quantities for nitrogen fertilizer applied per unit area, which is consistent not only with fertilizing best practice manuals, but also with the European Commission's standards for limiting nitrate pollution and for protecting areas within the network "Natura 2000".

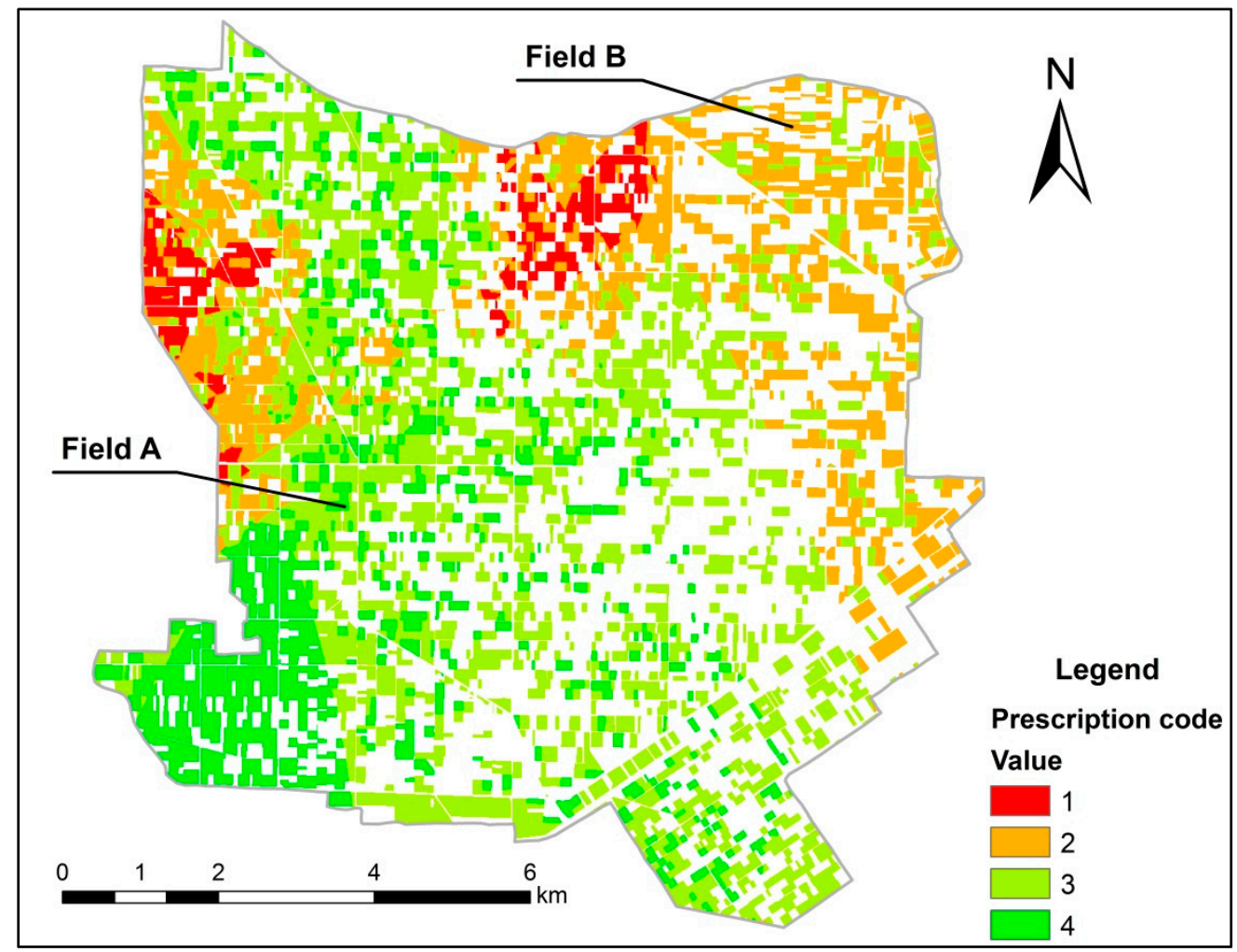

Figure 9. The spatial output of the "fertilizing type" module.

The evaluation of the "fertilizing type" module produced the map presented in Figure 9, which classifies the study area into four management zones. The consequent classes are sorted from lower to higher risk of soil degradation stemming from fertilizer use and are symbolized with red to green hues accordingly. Following, the classes were attributed with certain descriptive fertilizing recommendations 
given the numbers from one to four. The assignment was made with the contribution of experts in the field of soil fertility and nutrient kinetics and refers to the site-specific soil conditions of the study area. The fertilizing recommendations attached to each of the four codes are:

Prescription Code 1: Sites that fall into this category do not run any risk of acidification. Their $\mathrm{pH}$ is moderately alkaline, and only acidic fertilizers are proposed. Nitrogen fertilizers can be either in ammonium or nitrate forms. The fertilizer can be surface applied even in a single dose; however, it is recommended to incorporate it at two to three doses, in order to diminish gaseous or leaching losses.

Prescription Code 2: In the case of these sites, only acidic fertilizers that do not contain nitrate or urea nitrogen are proposed. They may well contain ammonium forms of nitrogen. Producers are encouraged to incorporate the fertilizer and apply it in at least two doses.

Prescription Code 3: In this case, the producer should avoid nitrate or urea fertilizers. The fertilizer can be applied on the surface of the field, but the overall application should concern at least three doses, distributed timely along with the crop needs.

Prescription Code 4: The application of all fertilizers is allowed. However, it is preferable not to apply acidic fertilizers due to increased risk of long-term reduction of the soil $\mathrm{pH}$. The measurement of soil acidity in those sites at regular intervals is essential. Furthermore, sprinkler irrigation systems should be avoided for diminishing leaching potential.

The prescription that is dominantly suggested across the interest area (51.0\% over the total area) is the third one followed by the second $(23.7 \%)$ and the fourth $(20.1 \%)$. The least suggested prescription is the first one, accounting for the $5.2 \%$ of the study area. Regarding the referenced Fields A and B, the module attributes different prescriptions, namely 4 and 2 accordingly (Figure 9). Further, the module suggests substituting the irrigation system in the case of Field A from sprinkler to a drip, yet for Field $\mathrm{B}$, it proposes the incorporation of fertilizer inputs, a practice that the producer has already adopted. In general, the module's results establish an illustrative zonal fertilizing prescription on the type, dose and time of fertilization across the study area. Indeed, along with the "fertilizing rate" module, past empirical treatment of fertilization on the region is replaced by a structured and methodical spatial approach to the calculation of the necessary fertilizing inputs. Through GIS mapping, the results of the system's runs are readily available to the end user, who in a single query can retrieve fertilizing prescriptions for any cotton field situated in the reference area.

The sensitivity analysis' results of "fertilizing rate" module, concerning ten of the fourteen input parameters whose value fields are continuous, are presented in Table 2. According to these results, the increase of five input parameters (soil inorganic nitrogen, clay content, mean soil temperature, quantity of irrigated water and inorganic nitrogen in irrigated water) resulted in diminishing the suggested average fertilizing rates across the study area. This finding is rather reasonable and expected for soil inorganic nitrogen, the quantity of irrigated water, inorganic nitrogen in irrigated water and the mean soil temperature, since these parameters affect solely the inputs of the nitrogen balance and indeed are positively correlated with those (Figure 6). In effect, the increase of their values tends to increase nitrogen inputs, driving the module to suggest less fertilizer in places where this occurs. 
Table 2. Results of Sensitivity Index I (dimensionless) for the "fertilizing rate" module.

\begin{tabular}{crcccc}
\hline \multirow{2}{*}{ No. } & \multirow{2}{*}{ Input parameters } & \multicolumn{4}{c}{$\%$ additive variation over the base model } \\
\cline { 3 - 6 } & & $\mathbf{5}$ & $\mathbf{1 0}$ & $\mathbf{1 5}$ & $\mathbf{2 0}$ \\
\hline 1 & Soil inorganic nitrogen & -0.81 & -1.57 & -2.21 & -2.69 \\
2 & Clay content & -4.32 & -10.06 & -16.01 & -21.82 \\
3 & pH & 5.38 & 10.57 & 10.85 & 10.95 \\
4 & Organic matter & -1.23 & -1.11 & 2.76 & 3.97 \\
5 & Soil moisture & 0.00 & 0.00 & 0.00 & 0.01 \\
6 & Mean soil temperature & -7.18 & -16.41 & -24.96 & -29.96 \\
7 & Mean rainfall & 7.19 & 12.09 & 12.68 & 13.90 \\
8 & Quantity of irrigated water & -0.65 & -1.20 & -1.70 & -2.21 \\
9 & Inorganic nitrogen in irrigated water & -0.88 & -1.67 & -2.17 & -2.49 \\
10 & Yield & 2.15 & 4.28 & 7.54 & 9.25 \\
\hline
\end{tabular}

Conversely, the clay content influences three parameters of the nitrogen balance (mineralization, leaching, denitrification), making the interpretation of the sensitivity analysis not so straightforward. The analysis' results showed that, according to the site-specific conditions of the study area, the additive variation in the values of clay percentage increases of nitrogen inputs (mineralization) may be indirect because of the increase in the water availability and further decreases leaching potential through reducing drainage conditions. When referring to the organic matter, an additive increase up to $10 \%$ increases nitrogen inputs, but a further increase diminishes them compared to the outputs (Table 2). This happens because the soil organic matter percentage drives both mineralization (input) and denitrification (output) processes. For small changes, the mineralization's products surpass the denitrification ones, while for larger variations, the opposite takes place.

The increase of the initial values of the parameters soil $\mathrm{pH}$, mean rainfall and yield potential resulted in increasing amounts of the proposed fertilizers. Finally, changes in the initial values of soil moisture did not substantially change the results of the module.

Classifying the input variables based on the results of the sensitivity analysis, the "fertilizing rate" module proved more sensitive to changes in soil temperature, clay content, rainfall, soil $\mathrm{pH}$, yield potential, organic matter content, soil inorganic nitrogen and "quality" and quantity of irrigation water, in the order reported.

The values in Table 3 refer to the area, as a percentage of the total study area, for which the fertilizing prescription code changed after the application of each variation $(5 \%, 10 \%$, etc.).

Table 3. Results of the sensitivity analysis for the "fertilizing type" module (percentage of the changes over the total study area).

\begin{tabular}{clcccc}
\hline \multirow{2}{*}{ No. } & \multirow{2}{*}{ Input parameters } & \multicolumn{4}{c}{ \% Additive variation over the base model } \\
\cline { 3 - 6 } & & $\mathbf{5}$ & $\mathbf{1 0}$ & $\mathbf{1 5}$ & $\mathbf{2 0}$ \\
\hline 1 & Clay content & 0.0 & 0.0 & 0.1 & 1.1 \\
2 & pH & 15.0 & 81.6 & 81.6 & 97.9 \\
3 & Mean rainfall & 0.0 & 43.8 & 63.5 & 63.5 \\
\hline
\end{tabular}


According to Table 3, the most important input variable, in terms of the sensitivity of this module, is soil $\mathrm{pH}$ followed by the precipitation and, finally, the clay content.

\section{Conclusions}

Both economic and environmental reasons, including compliance with directives of the European Commission, call for credible fertilization advisory, which should be based on objective scientific criteria. Towards this direction, space is essential and should not be overlooked in any fertilizing plan; especially if environmental conditions vary from place to place and affect decision making. The paper proposes a fuzzy GIS-based methodology for determining the appropriate rates and prescriptions for nitrogenous fertilizers in fields, in the form of both spatial and descriptive information. Through the developed spatial decision support system, the combination of various parameters (physical and anthropogenic) that affect and define fertilizing strategies is achieved. Further, any difficulties in the quantification of parameters that describe environmental processes were surpassed through the use of fuzzy logic concepts. The proposed system achieves the delineation of fertilizing management zones, based on specific criteria, expert knowledge and indigenous experience. Optionally, the intermediate system's results (e.g., mineralization, leaching potential, etc.) can be further obtained and evaluated. The parameters accounted for in the system can be enriched with new ones according to the special characteristics of an application area, whereas other variables can be overwritten by the user, increasing the system's flexibility. In addition, the evaluation scale of the system is defined by the availability and the spatial character of the input variables, which makes the system applicable both in the field and at the regional level. The system can be applied in other spring crops, under the prerequisite of adjusting the membership functions in the use and feeding the modules with proper input data, which in the case of the GIS modelling environment used, is proven to be straightforward.

Further research would include the application of the system to other agricultural areas, under different environmental and management conditions, which will control the system's consistency for reliable results. These results ought to be brought to the field level throughout at least three years of experimentation, for ascertaining the validity of the proposed fertilizer rates and suggestions. In situ work and testing would provide useful feedback regarding the weaknesses or limitations of the system, giving the opportunity for future amendments. Furthermore, the sensitivity analysis should be extended to include variations in more than one input variables in order to reveal correlations and regressions. Future planning will deal with the development of a user-friendly interface under an open source platform, combined with web GIS services.

\section{Acknowledgments}

The authors would like to acknowledge financial support from the State Scholarship Foundation of Greece. 


\section{Author Contributions}

Antonis Papadopoulos, Dionissios Kalivas and Thomas Hatzichristos contributed to the conception and design of this research. Antonis Papadopoulos performed the research, collected and analyzed the data, built, run and evaluated the system and wrote the manuscript. Dionissios Kalivas conducted the organization of the content. Antonis Papadopoulos and Dionissios Kalivas corrected the manuscript and addressed the comments of the reviewers. All authors read and approved the final manuscript.

\section{Conflicts of Interest}

The authors declare no conflict of interest.

\section{References and Notes}

1. European Commission, Council Directive 91/676/EEC concerning the protection of waters against pollution caused by nitrates from agricultural sources, 1991.

2. Ferguson, R.B.; Hergert, G.W.; Schepers, J.S.; Gotway, C.A.; Cahoon, J.E.; Peterson, T.A. Site-Specific Nitrogen Management of Irrigated Maize. Soil Sci. Soc. Am. J. 2002, 66, 544-553.

3. Tisdale, S.L.; Nelson, W.L.; Beaton, J.D.; Havlin, J.L. Soil Fertility and Fertilizers, 5th ed.; MacMillan Pub. Com.: New York, NY, USA, 1993.

4. Hirel, B.; Tétu, T.; Lea, P.J.; Dubois, F. Improving Nitrogen Use Efficiency in Crops for Sustainable Agriculture. Sustainability 2011, 3, 1452-1485.

5. Kersebaum, K.C.; Hecker, J-M.; Mirschel, W.; Wegehenkel, M. Modelling water and nutrient dynamics in soil - Crop systems: A comparison of simulation models applied on common data sets. In Modelling Water and Nutrient Dynamics in Soil-Crop Systems; Springer: Berlin, Germany, 2007; pp. 1-17.

6. Li, Y.; White, R.; Chen, D.; Zhang, J.; Li, B.; Zhang, Y.; Huang, Y.; Edis, R. A spatially referenced water and nitrogen management model (WNMM) for (irrigated) intensive cropping systems in the North China Plain. Ecol. Model. 2007, 203, 395-423.

7. Delgado, J.A.; Gross, C.M.; Lal, H.; Cover, H.; Gagliardi, P.; McKinney, S.P.; Hesketh, E.; Shaffer, M.J. A New GIS Nitrogen Trading Tool Concept for Conservation and Reduction of Reactive Nitrogen Losses to the Environment. Adv. Agron. 2010, 105, 117-171.

8. Benedikt, J.; Reinberg, S.; Riedl, L. A GIS application to enhance cell-based information modeling. Inf. Sci. 2002, 142, 151-160.

9. Mulla, D.J.; Schepers, J.S. Key processes and properties for site-specific soil and crop management. In The State of Site-Specific Management for Agriculture; American Society of Agronomy: Madison, WI, USA, 1997; pp. 1-18.

10. Ashraf, A.; Akram, M.; Sarwar, M. Fuzzy decision support system for fertilizer. Neural Comput. Appl. 2014, 25, 1495-1505.

11. Kweon, G. Delineation of site-specific productivity zones using soil properties and topographic attributes with a fuzzy logic system. Biosyst. Eng. 2012, 112, 261-277.

12. Assimakopoulos, J.H.; Kalivas, D.P.; Kollias, V.J. A GIS-based fuzzy classification for mapping the agricultural soils for N-fertilizers use. Sci. Total Env. 2003, 309, 19-33. 
13. Papadopoulos, A.; Kalivas, D.; Hatzichristos, T. Decision support system for nitrogen fertilization using fuzzy theory. Comput. Electron. Agric. 2011, 78, 130-139.

14. Kastanis, D. Soil Survey of the Plain of Kopaida; Land Resources Service. Hellenic Ministry of Agriculture Pub.: Athens, Greece, 1986.

15. Hellenic Statistical Authority. Annual Agricultural Statistical Survey. Areas under Cultivation: Provisional Results 2012; Hellenic Statistical Authority Pub.: Piraeus, Greece, 2014.

16. Oliver, M.A.; Webster, R. Kriging: a method of interpolation for geographical information systems. Int. J. Geogr. Inf. Syst. 1990, 4, 313-332.

17. Burrough, P.A.; McDonnell, R.A. Optimal interpolation using geostatistics. In Principles of Geographical Information Systems; Burrough, P.A., McDonnell, R.A., Eds.; Oxford University Press: Oxford, UK, 1998; pp. 132-161.

18. Lilburne, L.; Tarantola, S. Sensitivity analysis of spatial models. Int. J. Geogr. Inf. Sci. 2009, 23, 151-168.

19. Saltelli, A.; Tarantola, S.; Campolongo, F.; Ratto, M. Sensitivity Analysis in Practice. A Guide to Assessing Scientific Models; John Wiley and Sons Ltd.: New York, NY, USA, 2004.

20. Lenhart, T.; Eckhardt, K.; Fohrer, N.; Frede, H.G. Comparison of two different approaches of sensitivity analysis. Phys. Chem. Earth 2002, 27, 645-654.

21. Zhang, K.; Yang, D.; Greenwood, D.J.; Rahn, C.R.; Thorup-Kristensen, K. Development and critical evaluation of a generic 2-D agro-hydrological model (SMCR_N) for the responses of crop yield and nitrogen composition to nitrogen fertilizer. Agric. Ecosyst. Environ. 2009, 132, 160-172.

22. Antonopoulos, V.Z. Modelling of water and nitrogen balances in the ponded water and soil profile of rice fields in Northern Greece. Agric. Water Manag. 2010, 98, 321-330.

23. Pedrycz, W. Why triangular membership functions. Fuzzy Sets Syst.1994, 64, 21-30.

24. Zadeh, L.A. Fuzzy sets. Inf. Control 1965, 8, 338-353.

25. Cox, E. The Fuzzy Systems Handbook: A Practitioner's Guide to Building, Using, and Maintaining Fuzzy Systems; Academic Press Inc.: Waltham, MA, USA, 1994.

26. Wegehenkel, M.; Mirschel, W. Crop growth, soil water and nitrogen balance simulation on three experimental field plots using the Opus model-A case study. Ecol. Model. 2006, 190, 116-132.

27. Jeong, H.; Jang, T.; Seong, C.; Park, S. Assessing nitrogen fertilizer rates and split applications using the DSSAT model for rice irrigated with urban wastewater. Agric. Water Manag. 2014, 141, $1-9$.

28. Tsoukalas, L.H.; Uhrig, R.E. Fuzzy and Neural Approaches in Engineering; John Wiley and Sons, Inc.: New York, NY, USA, 1997; p. 587.

29. Masters, T. Practical Neural Network Recipes in C++; Academic Press: New York, NY, USA, 1993; p. 285.

30. Government Gazette, Issue No. 1195/B. Greek Action Plan for the Mitigation of Nitrates in Water Resources of Kopaida District; National Printing House: Athens, Greece, 2001.

31. Davatgar, N.; Neishabouri, M.R.; Sepaskhah, A.R. Delineation of site specific nutrient management zones for a paddy cultivated area based on soil fertility using fuzzy clustering. Geoderma 2012, 173-174, 111-118. 
32. Peralta, N.R.; Costa, J.L.; Balzarini, M.; Franco, M.C.; Córdoba, M.; Bullock, D. Delineation of management zones to improve nitrogen management of wheat. Comp. Electron. Agric. 2015, 110, $103-113$.

(C) 2015 by the authors; licensee MDPI, Basel, Switzerland. This article is an open access article distributed under the terms and conditions of the Creative Commons Attribution license (http://creativecommons.org/licenses/by/4.0/). 